\title{
A relação entre o criticismo do treinador e a ansiedade dos atletas: 0 papel do mindfulness e do autojulgamento
}

\author{
The relationship between coach criticism and athletes' anxiety: The role of \\ mindfulness and self-judgment
}

\author{
Rita Gama (1) \\ Marina Cunha $(1,2)$ \\ Cláudia Ferreira (2) \\ Ana Galhardo $(1,2)$ \\ Sara Oliveira (2)
}

(1) Instituto Superior Miguel Torga, Coimbra, Portugal

(2) Universidade de Coimbra, Centro de Investigação em Neuropsicologia e Intervenção Cognitiva e Comportamental (CINEICC), Portugal

Recebido: 20/07/2021; Revisto: 20/09/2021; Aceite: 21/10/2021.

\section{Resumo}

Contexto e Objetivo: Analisou-se de que forma a perceção dos atletas acerca das atitudes críticas do treinador teve efeito na ansiedade desportiva, explorando o papel dos traços de mindfulness e das atitudes de autocompaixão e de autojulgamento dos atletas. Métodos: A amostra incluiu 184 atletas adolescentes (150 rapazes e 34 raparigas), com idades entre os 12 e os 18 anos $(M=14,30 ; D P=1,96)$ que completaram um protocolo composto por quatro instrumentos de autorresposta que avaliaram a ansiedade desportiva, a perceção das atitudes críticas do treinador, o mindfulness, autocompaixão e autojulgamento. Resultados: A ansiedade desportiva demonstrou uma associação positiva com as atitudes críticas do treinador e com o autojulgamento, e uma associação negativa com o mindfulness. 0 mindfulness demonstrou uma associação negativa com o autojulgamento e com a perceção que os atletas têm acerca das atitudes críticas do treinador. O autojulgamento demonstrou-se positivamente associado com as atitudes críticas do treinador. O modelo preditor explicou 45\% da variância da ansiedade desportiva, sendo o mindfulness e o autojulgamento os únicos preditores. Os resultados da Path Analysis indicaram que o efeito das atitudes críticas do treinador na ansiedade desportiva foi totalmente mediado pelo mindfulness e pelo autojulgamento do atleta. Conclusões: Os dados sugerem que os atletas que percecionam mais atitudes críticas por parte do treinador, que têm mais atitudes de autojulgamento, e menos competências de autoconsciência e atenção plena, tendem a apresentar maiores níveis de ansiedade desportiva. O presente estudo teve um papel inovador ao evidenciar a forma específica como estas variáveis estão associadas, sugerindo que o desenvolvimento de competências de mindfulness e a diminuição de atitudes de autojulgamento podem ser úteis na diminuição do impacto das atitudes críticas do treinador na ansiedade competitiva.

Palavras-chave: Ansiedade desportiva; Perceção de atitudes críticas do treinador; Mindfulness; Autocompaixão; Autojulgamento.

\section{DI\&D | ISMT}

rpics@ismt.pt

https://rpics.ismt.pt distribuído sob a Licença Creative Commons Attribution, que permite uso, distribuição e reprodução sem restrições em qualquer meio, desde que o trabalho original seja devidamente citado.

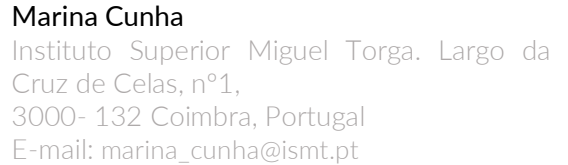




\begin{abstract}
Background and Aim: The sample included 184 adolescent athletes (150 boys and 34 girls), aged between 12 and 18 years old, who completed a protocol of self-report instruments assessing sports anxiety (SAS-2), the perception of coach's critical attitudes (APoCCAS), mindfulness (CAMM) and self-compassion (SCS). Method: The sample included 184 adolescent athletes (150 boys and 34 girls), aged between 12 and 18 years old, who completed a protocol of selfreport instruments assessing sports anxiety (SAS-2), the perception of coach's critical attitudes (APoCCAS), mindfulness (CAMM) and self-compassion (SCS). Results: Sports anxiety showed a positive association with critical coach attitudes and self-judgment and a negative association with mindfulness. Mindfulness revealed a negative association with selfjudgment and the athletes' perception of the coach's critical attitudes. Self-judgment was positively associated with the athletes' perception of the coach's critical attitudes. The regression model explained $45 \%$ of the variance in sports anxiety, with mindfulness and self-judgment being the only predictors. Path analysis results indicated that the athlete's mindfulness and self-judgment fully mediated the effect of the athletes' perception of the coach's critical attitudes on sports anxiety. Conclusions: Results suggest that athletes who perceive more critical attitudes from the coach, who have more self-judgmental attitudes, and fewer mindfulness skills tend to have higher levels of sports anxiety. The present study is innovative in showing how these variables are associated, suggesting that the development of mindfulness skills and the reduction of self-judgmental attitudes can help decrease the impact of the coach's critical attitudes on competitive anxiety.
\end{abstract}

Keywords: Sport anxiety; Athletes' perception of coach's critical attitudes; Mindfulness; Self-compassion; Self-judgment.

\title{
Introdução
}

Os momentos que antecedem os eventos competitivos provocam diversos estados emocionais que podem variar entre a realização pessoal e o equilíbrio emocional até estados mais negativos, como a preocupação, ansiedade e desânimo, geradores de comportamentos que influenciam o rendimento desportivo dos atletas (Santos et al., 2013).

A ansiedade é uma das reações emocionais mais importantes na atuação desportiva, sendo definida como um estado mental de inquietação e desordem (Machado et al., 2016), caracterizado pela presença de nervosismo, reatividade emocional ou excitação que provocam no indivíduo um estado emocional irrealista e desagradável (Bali, 2015). A ansiedade, que resulta da forma como o atleta perceciona o perigo real ou imaginário, é responsável pela sua adaptação a situações de perigo, tornando-se essencial a qualquer modalidade desportiva, uma vez que, num nível adequado se torna indispensável para o bom rendimento desportivo do atleta (Bali, 2015; Santos et al., 2013). Quando relacionada com a competição desportiva, a ansiedade é fortemente influenciada por fatores relacionados com o medo de falhar, medo das consequências sociais do seu desempenho, medo de se lesionar ou lesionar um adversário e o medo de não ser capaz de cumprir as funções necessárias à competição (Hasanah \& Refanthira, 2020). Desta forma, a ansiedade pode ser considerada uma reação emocional negativa quando a autoestima do atleta é ameaçada pela perceção que este tem de que a competição é um desafio que não consegue ultrapassar (Hasanah \& Refanthira, 2020). A ansiedade pode ser compreendida de duas formas distintas, ansiedadetraço e ansiedade-estado. A ansiedade-traço é considerada uma característica estável da personalidade (Machado et al., 2016), que predispõe o atleta a perceber a competição como ameaçadora e perigosa, despoletando níveis de ansiedade desproporcionais à situação (Weinberg \& Gould, 2017). A ansiedadeestado é descrita como um estado emocional transitório (Machado et al., 2016) que promove elevados níveis de nervosismo, preocupação e apreensão associados à excitação sentida (Weinberg \& Gould, 2017). 
Segundo Weinberg e Gould (2017), a ansiedade desportiva é influenciada por duas variáveis situacionais, a importância da competição e a incerteza relacionada com toda a conjuntura que envolve o mundo desportivo, sabendo que quanto maior a importância dada à competição ou a incerteza associada, maior será o nível de stress gerado. Quando o atleta tem dificuldade em lidar com a ansiedade, a sua capacidade adaptativa pode ser afetada. Neste caso, o atleta é tomado pela excitação e consequente incapacidade de se concentrar, o que diminui a sua competência de processamento de informação e lhe provoca respostas comportamentais mais lentas ou incorretas (Bali, 2015), que terão consequências diretas no seu rendimento desportivo.

A relação entre o treinador e o atleta constitui uma variável que tem um impacto na ansiedade competitiva (Ribeiro et al., 2016). De facto, a relação treinador-atleta de extrema importância já que permite ao treinador guiar o atleta, dar-Ihe apoio e fornecer-Ihe estratégias de orientação e suporte. Neste sentido, a relação deve ser mediada pela confiança, respeito, cooperação e compreensão mútua que contribuem para o desempenho e satisfação do atleta, visando, não só alcançar o melhor desempenho desportivo do atleta, mas também potenciar as competências profissionais do treinador (Jowett, 2007). Neste sentido, a relação entre treinador e atleta deve basear-se em princípios como a proximidade, o comprometimento e complementaridade (Jowett, 2011).

De facto, a literatura tem demonstrado que a relação treinador-atleta influencia não só a performance do atleta, como também o seu desenvolvimento psicossocial. O treinador é uma figura significativa para os atletas e as suas atitudes e comportamentos podem afetar a performance dos atletas e o seu bem-estar geral (Côté \& Fraser-Thomas, 2007; Davis \& Jowet, 2010). Apesar de a literatura sublinhar a importância de maior investigação na área da relação treinador-atleta (e.g., Poczwardowski et al., 2006), pouco se sabe acerca da experiência psicológica dos atletas e das suas perceções acerca de atitudes críticas dos seus treinadores (Siekanska et al., 2013), especialmente em adolescentes. Por exemplo, um estudo conduzido por Siekanska et al. (2013) mostrou que os atletas reconhecem que as atitudes excessivamente críticas por parte do treinador dificultam o seu desenvolvimento desportivo (Siekanska et al., 2013). Recentemente, também foi demonstrada uma associação entre a perceção que os atletas têm acerca de atitudes críticas do treinador e indicadores de psicopatologia numa amostra de atletas adultos (Oliveira et al., 2021a). No estudo citado, a perceção que os atletas têm acerca de atitudes críticas do treinador foi associada positivamente com as atitudes autocríticas do atleta (Oliveira et al., 2021a). Neste sentido, os atletas podem internalizar as críticas e julgamentos negativos do treinador, o que promove a autodepreciação, também denominado autocriticismo (Gilbert \& Irons, 2009; Oliveira et al., 2021a), no entanto esta associação ainda não foi testada em atletas adolescentes.

O autocriticismo é definido como um autojulgamento negativo, em que o sujeito demonstra uma resposta punitiva em relação aos seus erros, falhas ou características que possam provocar rejeição social (Gilbert et al., 2004). Desta forma, o autocriticismo pode ser definido como uma relação negativa do indivíduo com o seu eu perante erros ou fracassos associados a sentimentos de inadequação ou de inferioridade. Este processo de autoavaliação negativa pode também ser visto como um processo de regulação emocional na medida em que traduz a forma como a pessoa lida com as emoções difíceis de fracasso, inadequação ou desvalorização (Gilbert et al., 2004). Diversos estudos têm demonstrado uma associação entre o autocriticismo e o sofrimento emocional, desempenhando, assim, um fator de risco para o desenvolvimento de psicopatologia (e.g., Halamová et al., 2018). No contexto desportivo a maioria dos estudos são realizados em populações de adultos, por exemplo o estudo de Walton et al. (2020) concluiu 
que o autocriticismo constitui um preditor do stress psicológico para os atletas. Adicionalmente, PintoGouveia e Xavier (2010) demonstraram que o autocriticismo mantém uma relação positiva com a ansiedade competitiva, sendo um bom preditor da ameaça percebida gerada pela competição. Recentemente, foram ainda encontradas associações negativas entre o autocriticismo dos atletas e a sua qualidade de vida psicológica (Oliveira et al., 2021b). Um estudo conduzido em atletas adolescentes, demonstrou uma associação positiva entre autocriticismo e ansiedade, stress e depressão (Piló, 2013).

Por outro lado, o desenvolvimento de competências de mindfulness é uma alternativa para minorar os efeitos do autocriticismo sobre a ansiedade desportiva. Segundo Kabat-Zinn (1994, p. 20), mindfulness define-se como "prestar atenção de uma maneira particular, no momento presente e sem julgamento". Apesar da aparente incompatibilidade entre desporto e prática de mindfulness, esta estratégia tem vindo a ser cada vez mais utilizada como uma ferramenta essencial ao autocontrolo e foco no momento presente, o que tem implicações evidentes no desempenho desportivo e competitivo do atleta (Bühlmayer, et al, 2017; Dehghani et al, 2018). O estudo de Palmi e Solé (2016) concluiu que a prática de mindfulness produz efeitos no rendimento desportivo do atleta, através da promoção da aceitação, concentração, autorregulação emocional em situações de lesão desportiva, que proporcionam uma redução da ansiedade e stress-pré-competitivos. Outra investigação mostrou que níveis mais elevados de aceitação e mindfulness têm impacto nos resultados desportivos e estão frequentemente associados a menos sintomas de depressão e ansiedade (Pinto-Gouveia \& Xavier, 2010). No sentido oposto, este estudo revelou também que níveis mais baixos de aceitação e mindfulness e, consequentemente maior evitamento experiencial, estão mais frequentemente associados a sintomas de depressão e ansiedade nos atletas, o que exerce influência negativa nos resultados desportivos obtidos (Pinto-Gouveia \& Xavier, 2010). Estudos anteriores têm demonstrado o impacto positivo de programas baseados no mindfulness em contexto desportivo, tal como o programa Mindfulness-Based Soccer (MBSoccerP; Carraça, et al., 2018). Este programa demonstrou resultados positivos no aumento de mindfulness, flexibilidade psicológica, autocompaixão e estado de flow (estado de funcionamento ideal associado a picos de desempenho) que tem implicações não só no rendimento desportivo do atleta, mas também na sua vida fora do desporto. Paralelamente, foram desenvolvidos outros programas baseados no mindfulness como por exemplo o mindfulness sport performance enhancement (MSPE) e mindfulness meditation training for sport (MMTS). Estes programas desenvolvidos para aplicação em contexto desportivo pretenderam promover a atenção ao momento presente e a aceitação dos estados internos de uma forma não julgadora, os quais têm revelado resultados positivos nos atletas (Baltzell \& Akhtar, 2014; Josefsson et al., 2019; Kaufman et al., 2009). O programa MSPE demonstrou resultados positivos ao nível do flow, mindfulness, e aspetos relacionados com a confiança desportiva. Por sua vez, o programa MMTS revelou melhorias no mindfulness não demonstrando alterações nas pontuações médias referentes a emoções positivas e negativas dos atletas em estudo.

Relativamente à autocompaixão, vão também surgindo estudos que comprovam a associação negativa entre autocompaixão e a ansiedade e rendimento desportivo (e.g., Ferguson et al., 2014; Mosewich et al., 2011, 2013).

Neff (2003) descreve a autocompaixão como sendo constituída por três componentes interrelacionados: capacidade de ser compreensível consigo próprio e não excessivamente autocrítico (calor/compreensão vs. autocriticismo), capacidade de entender as suas experiências como fazendo parte de uma condição humana comum e não como experiências isoladas (condição humana vs. isolamento) e consciência dos 
próprios pensamentos e sentimentos dolorosos sem excessiva sobreidentificação (mindfulness vs. sobreidentificação).

A título de exemplo, indicamos o estudo de Killham et al. (2018), que teve como objetivo analisar se a autocompaixão e o autocriticismo exercem influência no desempenho desportivo percebido em mulheres atletas. De acordo com estes dados, a autocompaixão encontra-se relacionada com a perceção de um melhor rendimento desportivo e o autocriticismo relacionado com níveis mais baixos de rendimento percebido. Assim, o mesmo estudo concluiu que a autocompaixão, para além de desempenhar um papel fundamental na perceção do rendimento desportivo, auxilia ainda a atleta a lidar com o autocriticismo no desporto. Esta forma compreensiva, calorosa e autoconsciente do indivíduo se relacionar consigo próprio, ou de autorregular as suas emoções, pode ser promovida pelo relacionamento que o atleta estabelece com o seu treinador, exercendo os treinadores um papel importante (Frentz, 2019).

Em síntese, a revisão da literatura evidenciou que variáveis como a relação treinador-atleta, estratégias de regulação emocional dos atletas (como uma atitude autocompassiva, uma atitude crítica e autojulgadora e uma atitude de autoconsciência e de aceitação plena) podem ter um papel relevante na ansiedade desportiva. De notar que as atitudes excessivamente críticas do treinador podem envolver o atleta numa espiral de autocrítica e ruminação (Frentz, 2019). Na verdade, o autocriticismo, a autoculpa e a ruminação são expressões emocionais frequentemente relatadas pelos atletas perante as experiências desportivas de fracasso (Ferguson et al., 2014, 2015; Mosewich et al., 2013). Inversamente, quando o treinador procura entender os problemas e preocupações dos atletas, estes tendem a demonstrar uma maior autocompaixão traduzida por uma atitude mais tolerante e sensível ao seu próprio sofrimento (Frentz, 2019). No entanto, a maioria das investigações tem sido conduzida em populações de adultos, e o estudo destas variáveis é escasso em amostras de adolescentes.

Assim, o objetivo do presente estudo é analisar de que forma é que a perceção dos atletas acerca das atitudes críticas do treinador tem efeito na ansiedade desportiva, explorando o papel dos traços de mindfulness e das atitudes de autocompaixão e de autojulgamento em jovens atletas. Especificamente, hipotetiza-se que atletas que percecionam mais atitudes críticas por parte do treinador apresentem maiores níveis de ansiedade desportiva, através de maiores níveis de autojulgamento e menores traços de mindfulness e de autocompaixão.

\section{Método}

\section{Participantes}

A amostra do presente estudo foi constituída por 184 atletas de nacionalidade Portuguesa, dos quais 150 eram do sexo masculino (81,5\%) e 34 do sexo feminino (18,5\%), com idades compreendidas entre os $12 \mathrm{e}$ os 18 anos de idade $(M=14,30 ; D P=1,96)$. No que respeita à modalidade praticada, 143 atletas praticavam Futebol, 11 praticavam Judo, 2 praticavam Ténis, 14 praticavam Patinagem Artística e 14 praticavam Natação. Relativamente à escolaridade, os atletas apresentaram uma média de 8,87 anos de escolaridade $(D P=1,89)$. No que se refere à prática da modalidade, o tempo médio para a prática da mesma variou entre 1 e 156 meses ( $M=66,33$; $D P=41,36$ ), ainda que se tenham verificado alguns dados omissos respeitantes a esta variável. Relativamente, ao tempo em que o atleta se encontra no seu atual clube, oscilou entre 1 e 132 meses $(M=43,60 ; D P=31,38)$ e o número de treinos por semana variou entre 
1 e 6 meses $(M=3,09 ; D P=0,71)$. $O$ tempo que o atleta treina com o atual treinador oscilou entre $1 \mathrm{e}$ 156 meses $(M=25,52 ; D P=26,23)$, sabendo que 136 dos 184 atletas já foram treinados por outro treinador.

\section{Instrumentos}

\section{Sport Anxiety Scale-2 (SAS-2)}

A SAS-2 (Escala de Ansiedade no Desporto - 2; Cruz \& Gomes, 2007) permite verificar as diferenças individuais inerentes à ansiedade somática e às duas dimensões da ansiedade cognitiva (preocupação e perturbação da concentração). A ansiedade somática diz respeito aos indicadores fisiológicos, nomeadamente ao funcionamento dos músculos e do estômago dos atletas (e.g., "Sinto um nó no estômago"). A preocupação diz respeito à possibilidade de o atleta atingir um rendimento inferior ao expectável, e ainda às consequências negativas que este rendimento menos positivo possa acarretar para si (e.g., "Preocupa-me que vá jogar/competir mal)". A perturbação da concentração refere-se à incapacidade de o atleta estar concentrado nas tarefas que se propõe a realizar (e.g., "É difícil concentrarme no que tenho de fazer"). Dividido pelas subescalas ansiedade somática, preocupação e perturbação da concentração, o instrumento é constituído por 15 itens, cotado através de uma escala de Likert de cinco pontos que variam entre 0 ( $N a d a$ ) e 4 (Muito). O score de cada subescala é calculado através do somatório de todos os itens que a constituem e o score total da ansiedade competitiva é obtido através do somatório de todas as subescalas. As subescalas ansiedade somática, preocupação e perturbação de concentração apresentam valores de alfa de Cronbach coincidentes com uma boa consistência interna $(\alpha=0,85 ; \alpha=$ 0,86 e $\alpha=0,74$, respetivamente). No presente estudo, o valor de alfa de Cronbach revelou boa consistência interna para as subescalas que constituem o instrumento ( $\alpha=0,85$ para a subescala ansiedade somática, $\alpha=0,89$ para a subescala preocupação e $\alpha=0,81$ para a subescala perturbação de concentração), assim como para a escala total $(\alpha=0,90)$, a qual foi utilizada no presente estudo tendo em consideração os nossos objetivos.

\section{Athletes' Perceptions of Coach-Related Critical Attitudes Scale (APoCCAS-A)}

A APoCCAS-A (Escala sobre a Perceção dos Atletas acerca de Atitudes Críticas do Treinador; Oliveira et al., 2021a) é composta por dez itens que avaliam a perceção que o atleta tem acerca das atitudes críticas do seu treinador [e.g., "Percebo que o(a) meu(minha) treinador(a) fica ansioso(a) e/ou zangado(a) quando eu falho"]. Trata-se de instrumento unidimensional, cujos itens são cotados através de uma escala de Likert de cinco pontos de 1 (Discordo completamente) a 5 (Concordo completamente). O score é obtido através do somatório de todos os itens, tendo em conta que scores mais elevados indicam uma maior perceção de atitudes críticas dos treinadores. A presente escala apresenta uma boa consistência interna $(\alpha=0,90)$ (Oliveira et al., 2021a). Na nossa amostra o valor de $\alpha=0,87$ é indicador de boa consistência interna.

\section{Child and Adolescent Mindfulness Measure (CAMM)}

A CAMM (Medida de Mindfulness para Crianças e Adolescentes; Cunha et al., 2013a) pretende avaliar as competências de mindfulness (e.g., "Afasto de mim pensamentos que me desagradam). É composta por dez itens, sendo que a cotação é realizada através de uma escala de Likert de cinco pontos de 0 (Nunca) a 4 (Sempre). Trata-se de uma escala unidimensional em que o score total é resultado do somatório de todos os itens. Todos os itens são invertidos, sendo que quanto maior a pontuação total da escala, mais competências de mindfulness o jovem apresenta. Através do alfa de Cronbach, foi possível calcular a 
consistência interna da escala, que se revelou aceitável ( $\alpha=0,80$ ) (Cunha et al., 2013a). No presente estudo o valor de alfa de Cronbach obtido $(\alpha=0,82)$ é demonstrativo de boa consistência interna.

\section{Self Compassion Scale (SCS-A)}

A SCS-A (Escala de Autocompaixão para Adolescentes; Cunha et al., 2013b, 2016) permite medir a autocompaixão, ou seja, a capacidade de se sentir empático e compreensivo consigo mesmo em situações de sofrimento (e.g., "Tento ser afetuoso(a) comigo próprio(a) quando estou a sofrer"). É constituída por 26 itens distribuídos por seis subescalas: Autobondade (e.g., "Tento ser afetuoso(a) comigo próprio(a) quando estou a sofrer"); Isolamento (e.g., "Quando penso acerca das minhas inquietações e defeitos, sinto-me mais à parte e desligado(a) do resto do mundo"); Humanidade Comum (e.g., "Quando estou "em baixo" lembrome que existem muitas outras pessoas no mundo que se sentem como eu"); Autocrítica e.g., "Quando passo por tempos difíceis tenho tendência a ser muito exigente e duro(a) comigo mesmo(a)"); Mindfulness (e.g., "Quando alguma coisa dolorosa acontece tento ter uma visão equilibrada da situação") e Sobreidentificação [e.g., "Quando me sinto "em baixo" tenho tendência a ficar agarrado(a) e a ficar obcecado(a) com tudo aquilo que está errado"]. A cotação é realizada segundo uma escala de Likert de 5 pontos que varia de 1 (Quase nunca) a 5 (Quase sempre). O score de cada subescala é obtido através da média das pontuações obtidas em cada um dos itens. O score total da escala é o resultado da média das pontuações das subescalas, após cotação inversa das subescalas Isolamento, Autocrítica e Sobreidentificação. Pontuações mais altas remetem para maiores níveis de autocompaixão. A escala total apresenta um coeficiente de alfa de Cronbach indicador de uma boa consistência interna $(\alpha=0,85)$. Verifica-se uma consistência interna adequada para as dimensões que constituem a escala ( $\alpha=0,75$ na subescala Calor/Compreensão; $\alpha=0,75$ na subescala Isolamento; $\alpha=0,71$ na subescala Condição Humana; $\alpha=0,77$ na subescala Autocrítica; $\alpha=0,70$ na subescala Mindfulness; e $\alpha=0,73$ para a subescala Sobreidentificação). No presente estudo utilizámos a solução de dois fatores, respetivamente, as dimensões positivas (Autobondade, Humanidade Comum e Mindfulness) e as dimensões negativas (Autocrítica, Isolamento, Sobreidentificação). Ambos os fatores, designados por autocompaixão e autojulgamento, revelaram uma boa consistência interna (alfa de Cronbach foi de 0,89 e de 0,92, respetivamente).

\section{Procedimentos Metodológicos}

O presente estudo foi submetido à aprovação da Comissão de Ética do Instituto Superior Miguel Torga, tendo recebido parecer favorável (CE-P01-21).

Uma vez definidos os instrumentos necessários à elaboração do protocolo, foram solicitadas as autorizações para utilização das escalas aos respetivos autores.

Para recrutamento dos participantes, foram contactados alguns clubes desportivos da zona Centro de Portugal, tendo sido endereçados os respetivos pedidos de autorização para recolha de dados.

Uma vez que a amostra é constituída por adolescentes foram formulados e entregues os consentimentos informados dirigidos aos pais/tutores legais, onde foi facultada informação relativa ao estudo, bem como à confidencialidade dos dados obtidos. Tendo em conta a situação pandémica atual, alguns consentimentos foram enviados via e-mail. Após o preenchimento dos consentimentos informados, foi dado início à recolha dos dados através da administração do protocolo presencialmente ou online, sendo que a maioria foi preenchida em papel, e apenas 33 questionários tiveram preenchimento online. 


\section{Procedimentos Analíticos}

Os procedimentos estatísticos foram realizados através dos softwares de análise estatística Statistical Package for the Social Sciences (IBM SPSS Statistics) versão 27 e Analysis of Moment Structures (IBM SPSS AMOS) versão 22.

O tamanho da amostra foi, inicialmente, calculado tendo em consideração o número de parâmetros do modelo a testar. Sendo assim, seriam necessários no mínimo 140 sujeitos. Para caracterização sociodemográfica da amostra através de estatística descritiva foram calculadas as médias e respetivos desvios padrão para as variáveis contínuas e as frequências para as variáveis categoriais.

Para averiguação das diferenças dos valores médios em função do sexo, foi utilizado o teste $t$ de Student para amostras independentes e o $d$ de Cohen para analisar o tamanho do efeito.

Os valores de assimetria $(S k)$ e curtose $(K u)$ foram examinados para testar a distribuição normal das variáveis em estudo $(|S k|<3$; $|K u|<8$; Kline, 2005).

Para análise das associações estabelecidas entre as variáveis em estudo foram calculados os coeficientes de correlação de Pearson. A interpretação do tamanho das correlações seguiu a classificação de Marôco (2018) que distingue: correlação fraca (valores de $r<0,25)$, correlação moderada $(0,25 \leq r<0,50)$, correlação forte $(0,50 \leq r<0,75)$ e correlação muito forte $(r \geq 0,75)$.

A consistência interna dos instrumentos utilizados neste estudo foi avaliada através da análise do alfa de Cronbach, tendo sido utilizados como valores de referência os índices indicados por Pestana e Gageiro (2008), tendo em conta que os mesmos autores defendem que um valor de alfa inferior a 0,60 é indicador de uma consistência interna inadmissível, entre 0,60 e 0,70 a consistência interna é fraca, entre 0,70 e 0,80 a consistência interna é razoável, entre 0,80 e 0,90 uma consistência interna é boa e, se o alfa superior a 0,90 a consistência interna é considerada muito boa. Para as análises realizadas foi considerada a significância estatística com um valor de $p$ igual ou inferior a 0,05 (Marôco, 2018).

A análise de regressão múltipla foi calculada usando variáveis de regulação emocional (mindfulness, autocompaixão e autojulgamento) para predizer a ansiedade desportiva. Para esse efeito, foram analisados os pressupostos base, como a distribuição normal das variáveis e análise de multicolinearidade (Tabachnick \& Fidell, 2013). A regressão múltipla foi realizada como uma análise de suporte na escolha das variáveis a colocar no modelo de mediação a testar.

As análises de trajetórias (path analyses) foram conduzidas através do AMOS 22,0. Este tipo de modelo de equação estrutural pretende analisar a existência de efeitos diretos e indiretos entre diferentes variáveis (endógenas e exogéneas), controlando a presença de erros (Kline, 2005). Tendo em conta os objetivos do estudo, foi testado o efeito da perceção dos atletas acerca de atitudes críticas do treinador (APoCCAS; variável exógena) na ansiedade desportiva (SAS-2; variável endógena), examinado o efeito mediador do mindfulness (CAMM) e do autojulgamento (SCS-autojulgamento) nesta associação (variáveis endógenas mediadoras). O método de Máxima Verossimilhança foi utilizado para averiguar a significância dos coeficientes da via (path) e uma série de testes que visam calcular a qualidade do ajustamento do modelo ( $\chi^{2}$, CFI, TLI e SRMR; Hu \& Bentler, 1999). A adequação do modelo foi examinada tendo em consideração os seguintes índices de ajustamento: Qui-Quadrado $\left(x^{2}\right)$, que quando não é significativo indica um bom ajustamento dos dados; o Qui-quadrado normalizado (CMIN/gl), que se revela aceitável se apresentar um valor inferior a 5; o SRMR (Standardised Root Mean Square Residual) que deverá ser inferior a 0,08 e o CFI 
(Comparative Fit Index) e TLI (Tucker Lewis Index), que indicam um bom ajustamento se os valores forem superiores a 0,90 (Hu \& Bentler, 1999).

Os efeitos diretos, indiretos e totais estabelecidos entre as variáveis foram testados através do QuiQuadrado e do método Bootstrap resampling com 5.000 amostras e com um intervalo de confiança (IC) corrigido de 95\% (bias corrected confidence intervals). Nesta análise, considera-se que o efeito é estatisticamente significativo quando o IC não inclui o zero (Kline, 2005) e quando o $p$ é inferior a 0,05 (Tabachnick \& Fidell, 2013).

\section{Resultados}

\section{Análise Preliminar dos Dados}

O pressuposto da normalidade da distribuição das variáveis foi confirmado ( $|S k|<3$ e $|K u|<8$; Kline, 2005). De facto, os valores de assimetria variaram entre -0,55 (CAMM) e 0,78 (SAS-2), e os valores de curtose entre $-0,53$ (SCS-autojulgamento) e 0,33 (SAS-2).

\section{Análise Descritiva}

Na Tabela 1 são apresentados os resultados médios das variáveis em estudo, obtidos na amostra total, bem como a comparação dos resultados em função do sexo.

\section{Tabela 1}

Análise Descritiva das Escalas em Função do Sexo

\begin{tabular}{lccccccc}
\hline & $\begin{array}{c}\text { Total } \\
\text { Medidas }\end{array}$ & $M=184)$ & $\begin{array}{c}\text { Rapazes } \\
(n=34)\end{array}$ & $\begin{array}{c}\text { Raparigas } \\
(n=150)\end{array}$ & $t$ & $p$ & $d$ \\
\cline { 2 - 5 } Ansiedade desportiva & $28,49 \pm 7,66$ & $28,09 \pm 7,56$ & $30,26 \pm 7,94$ & $-1,50$ & 0,135 & 7,63 \\
Atitudes críticas do treinador & $22,92 \pm 6,99$ & $22,85 \pm 7,01$ & $23,24 \pm 7,02$ & $-0,29$ & 0,774 & 7,01 \\
Mindfulness & $28,47 \pm 6,70$ & $28,61 \pm 6,55$ & $27,82 \pm 7,40$ & $-0,62$ & 0,536 & 6,71 \\
Autocompaixão & $2,87 \pm 0,71$ & $2,91 \pm 0,69$ & $2,70 \pm 0,80$ & 1,56 & 0,163 & 0,71 \\
Autojulgamento & $3,37 \pm 2,47$ & $7,29 \pm 2,38$ & $7,73 \pm 2,87$ & $-0,94$ & 0,347 & 2,47 \\
\hline
\end{tabular}

Nota. A Ansiedade desportiva foi avaliada através da Sport Anxiety Scale-2; as atitudes críticas do treinador foram avaliadas através da Athletes' Perceptions of Coach-Related Critical Attitudes Scale; o mindfulness foi avaliado através da Child and Adolescent Mindfulness Measure; e a autocompaixão e autojulgamento foram avaliados através da Self Compassion Scale.

Não houve diferenças significativas entre rapazes e raparigas para a ansiedade desportiva $\left(t_{(182)}=-1,50 ; p\right.$ $=0,135)$, perceção dos atletas acerca de atitudes críticas do treinador $\left(t_{(182)}=-0,29 ; p=0,774\right)$, mindfulness $\left(t_{(182)}=0,54 ; p=0,536\right)$, autocompaixão $\left(t_{(182)}=1,64 ; p=0,103\right)$ e autojulgamento $\left(t_{(182)}=-0,94 ; p=0,347\right)$.

\section{Análise Correlacional}

Os valores dos coeficientes de correlação de Pearson estão indicados na Tabela 2. 
Tabela 2

Coeficientes de Correlação de Pearson

\begin{tabular}{lllll}
\hline Instrumentos de medida & 1 & \multicolumn{1}{c}{2} & \multicolumn{1}{c}{3} & \multicolumn{1}{c}{4} \\
\hline 1. Ansiedade desportiva & - & & & \\
2. Atitudes críticas do treinador & $0,35^{* * *}$ & - & & \\
3. Mindfulness & $-0,66^{* * *}$ & $-0,38^{* * *}$ & - & - \\
4. Autocompaixão & $-0,02$ & $-0,11$ & 0,07 & - \\
5. Autojulgamento & $0,56^{* * *}$ & $0,31^{* * *}$ & $-0,70^{* * *}$ & $-0,13$ \\
\hline
\end{tabular}

Nota. $N=184$. A Ansiedade desportiva foi avaliada através da Sport Anxiety Scale-2; as atitudes críticas do treinador foram avaliadas através da Athletes' Perceptions of Coach-Related Critical Attitudes Scale; o mindfulness foi avaliado através da Child and Adolescent Mindfulness Measure; e a autocompaixão e autojulgamento foram avaliados através da Self Compassion Scale. ${ }^{*} p<0,05 .{ }^{* * *} p<0,001$

Todos os resultados (Tabela 2) revelaram uma correlação estatisticamente significativa entre as variáveis em estudo, à exceção da autocompaixão. A ansiedade desportiva mostrou uma correlação positiva moderada com as atitudes críticas do treinador, uma correlação positiva forte com o autojulgamento e uma correlação negativa forte com o mindfulness. O mindfulness revelou uma correlação negativa forte e moderada com o autojulgamento e a perceção de atitudes críticas do treinador, respetivamente. $O$ autojulgamento demonstrou uma correlação positiva e moderada com a perceção de atitudes críticas do treinador. Por último, o fator da autocompaixão não se mostrou associado a qualquer outra variável.

A idade não evidenciou qualquer correlação significativa com a ansiedade desportiva ( $r=-0,04 ; p=0,557$ ), com as atitudes críticas do treinador $(r=-0,01 ; p=0,860)$, com o mindfulness $(r=-0,03 ; p=0,734)$, com a autocompaixão ( $r=-0,08 ; p=0,296)$ ou com o autojulgamento $(r=0,12 ; p=0,098)$.

\section{Modelo Preditor da Ansiedade Desportiva}

Conduzimos uma análise de regressão múltipla utilizando os processos de mindfulness, autojulgamento e autocompaixão para prever a ansiedade desportiva.

O modelo foi responsável por $45 \%$ da variância $\left(F_{(180,3)}=50,18 ; p<0,01\right)$. O mindfulness e o autojulgamento surgiram como os melhores preditores de ansiedade no desporto $(\beta=-0,52 ; p<0,01$ e $\beta$ $=0,20 ; p=0,011$, respetivamente). A autocompaixão $(\beta=0,4 ; p=0,439)$ não foi um preditor significativo.

\section{Análise de trajetórias (Path analysis)}

Através de uma análise de trajetórias (path analysis) foi testada a hipótese de que a perceção dos atletas acerca de atitudes críticas do treinador (APoCCAS) poderá ter um efeito na ansiedade desportiva (SAS-2) através do mindfulness (CAMM) e do autojulgamento (SCS-autojulgamento) (Figura 1). A opção pelo estudo deste modelo foi baseada na análise de correlações, a qual mostrou que a variável autocompaixão não se associou significativamente com as restantes variáveis em estudo. Paralelamente, os resultados da regressão apoiaram os resultados obtidos nas correlações, mostrando apenas o efeito preditivo do mindfulness e do autojulgamento na ansiedade desportiva. Assim, estes resultados apoiam a opção da não inclusão da autocompaixão no modelo a testar. Por último, apesar da idade poder ser uma variável importante no modelo de desenvolvimento desportivo, verificou-se que a mesma não se correlacionava 
significativamente com qualquer das variáveis em estudo. Neste sentido, optou-se por não controlar o efeito da idade no modelo mediacional.

O modelo foi testado através de um modelo saturado composto por 14 parâmetros. No entanto, a relação entre a APoCCAS e a SAS-2 não se revelou significativa ( $b_{\text {APocCAS }}=0,12 ; S E_{b}=0,06 ; Z=1,85 ; p=0,06$ ), pelo que esta ligação foi eliminada, e o modelo foi reajustado.

\section{Figura 1}

Modelo de Trajetórias

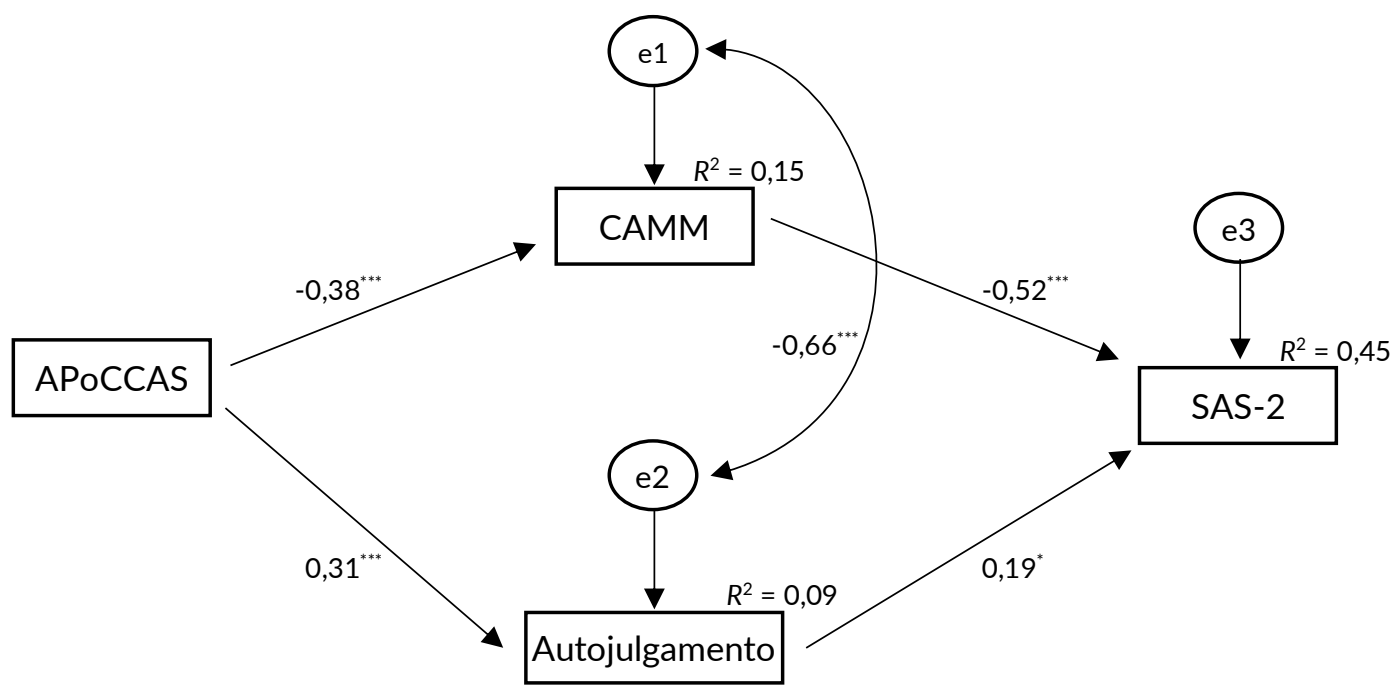

Nota. Os valores apresentados são coeficientes de regressão estandardizados. APoCCAS = Athletes' Perceptions of Coach-Related Critical Attitudes Scale; CAMM = Child and Adolescent Mindfulness Measure; da Sport Anxiety Scale-2; o Autojulgamento foi avaliado através da Self Compassion Scale. SAS-2 = Sport Anxiety Scale-2.

${ }^{*} p<0,05 .{ }^{* * *} p<0,001$

O modelo final revelou explicar $45 \%$ da variância da ansiedade desportiva (SAS-2), no qual todas as vias apresentaram significância estatística (Figura 1). Este modelo apresentou $1 \mathrm{gl}$ e uma excelente adequação aos dados empíricos $\left[\mathrm{X}^{2}(1)=3,39 ; p=0,066 ; \mathrm{CMIN} / \mathrm{gl}=3,39 ; \mathrm{TLI}=0,95 ; \mathrm{CFI}=0,99 ; \mathrm{SRMR}=0,292 ; p=\right.$ 0,128; (IC95\% = 0,00 - 0,26)]. A perceção dos atletas acerca de atitudes críticas do treinador revelou um efeito direto sobre o mindfulness de -0,38 ( $\left.b_{\text {APoCCAS }}=-0,37 ; S E_{b}=0,07 ; Z=-5,61 ; p<0,01\right)$ e de 0,31 sobre o autojulgamento $\left(b_{\mathrm{APoCCAS}}=0,11 ; S E_{\mathrm{b}}=0,03 ; Z=4,34 ; p<0,01\right)$. $O$ mindfulness revelou um efeito direto na ansiedade desportiva de $-0,52\left(b_{\text {CAMM }}=-0,60 ; S E_{\mathrm{b}}=0,09 ; Z=-6,85 ; p<0,01\right)$ e o autojulgamento revelou um efeito direto de 0,19 ( $b_{\text {scs_autojulgamento }}=0,60 ; S E_{\mathrm{b}}=0,24 ; Z=2,51 ; p=0,012$ ) na ansiedade desportiva. A análise dos efeitos indiretos demonstrou que a perceção dos atletas acerca de atitudes críticas do treinador apresenta uma associação indireta de 0,24 (IC95\% $=0,16$ a 0,33) na ansiedade desportiva. Em síntese, os resultados obtidos mostraram que o efeito da perceção dos atletas acerca de atitudes críticas dos treinadores na ansiedade desportiva é totalmente mediado pelo mindfulness e pelo autojulgamento $(\beta=0,26 ;$ IC95\% $=0,17$ a 0,35$)$. 


\section{Discussão}

O presente estudo pretendeu testar a associação entre a perceção dos atletas acerca de atitudes críticas do treinador e a ansiedade desportiva de atletas adolescentes, através do mindfulness e do autojulgamento.

Os resultados das análises descritivas demonstraram não existir diferenças estatisticamente significativas entre rapazes e raparigas em qualquer das variáveis em estudo. Relativamente aos resultados das correlações, a ansiedade desportiva demonstrou uma associação positiva com as atitudes críticas do treinador, com o autojulgamento e uma associação negativa com o mindfulness. Por sua vez, o mindfulness demonstrou uma associação negativa com o autojulgamento, ansiedade desportiva e a perceção que os atletas têm acerca das atitudes críticas do treinador. $O$ autojulgamento demonstrou-se negativamente associado com as atitudes críticas do treinador. Por outro lado, a variável autocompaixão não mostrou associar-se significativamente com as restantes variáveis em estudo.

Na globalidade, os resultados encontrados vão ao encontro de estudos anteriores que demonstram que atitudes excessivamente críticas por parte de figuras significativas, exercem influência na saúde mental dos adolescentes (Brewin et al., 1996; Frazer et al., 2018), encontrando-se intimamente ligadas a sintomas de depressão e ansiedade (McLeod et al., 2007; Wood et al., 2003). Existem estudos que comprovam que as atitudes excessivamente críticas por parte de figuras significativas bem como a desvalorização dos sentimentos e comportamentos do indivíduo, podem influenciar a regulação emocional e promover atitudes autocríticas (Koestner et al., 1991; Lee et al., 2015), sabendo que a autocrítica é descrita por alguns autores como o resultado da internalização das críticas dos outros (Brewin et al., 1996). Assim, tendo em consideração que o treinador representa uma figura significativa para os atletas, com impacto no seu desenvolvimento (Norman \& French, 2013), e que o criticismo por parte de figuras significativas pode promover atitudes autocríticas por parte dos indivíduos, estes resultados suportam dados anteriores, como por exemplo os resultados obtidos no estudo de Oliveira et al. (2021a), que demonstrou que atletas que percecionam mais atitudes críticas por parte do treinador parecem apresentar maiores níveis de autocriticismo. No entanto, o estudo citado anteriormente foi conduzido em atletas adultos, pelo que os nossos dados apesar de irem ao encontro dos anteriores, adicionam novas evidências à literatura demonstrando as mesmas associações na população adolescente. Relativamente à associação entre autojulgamento e ansiedade desportiva, mais uma vez, os nossos dados estão em concordância com um estudo de Gaetano et al. (2015) que defende que o autojulgamento dos atletas se associa a uma diminuição da autoestima e o aumento da ansiedade associada ao desporto em jovens atletas.

Paralelamente, a associação negativa entre a ansiedade desportiva e o mindfulness é corroborada por estudos anteriores, tais como o estudo de Scott-Hamilton et al. (2016) que revelou que atletas maiores de 16 anos de idade que usufruíram de um programa de intervenção baseado no mindfulness demonstraram uma diminuição da ansiedade desportiva e pessimismo relacionado com o desporto, quando comparados com o grupo de participantes que não foi alvo de intervenção. Por outro lado, um estudo conduzido por Spínola et al. (2019) concluiu que o mindfulness não se associou significativamente com a ansiedade competitiva em jovens atletas, contrariamente aos resultados obtidos no presente estudo. Assim, investigações futuras poderão esclarecer a significância da associação entre estas variáveis.

Relativamente à associação entre o mindfulness e a perceção das atitudes críticas do treinador, que seja do nosso conhecimento, não existem estudos prévios que suportem os resultados obtidos, destacando o caráter inovador do presente estudo que demonstra que atletas que percecionam mais atitudes críticas 
por parte do treinador tendem a apresentar menores níveis de mindfulness. Por fim, não foram encontradas associações significativas entre a autocompaixão e as restantes variáveis em estudo. Estudos anteriores conduzidos com atletas adolescentes do sexo feminino demonstraram que a autocompaixão se associou negativamente a outcomes negativos (e.g., ansiedade, medo de falhar; Mosewich et al., 2011). Assim, seria pertinente continuar a estudar o papel da autocompaixão em atletas jovens.

Os resultados da regressão mostraram que apenas o mindfulness e o autojulgamento foram variáveis preditoras da ansiedade competitiva, reforçando a ausência de significância da autocompaixão na predição da ansiedade desportiva. Apesar de, nas últimas décadas, terem sido demonstrados efeitos positivos da compaixão nos atletas (Baltzell et al., 2020), são ainda escassos os estudos relativamente a esta competência. De acordo com um estudo conduzido por Neely et al. (2009), apesar da autocompaixão se demonstrar útil na redução das consequências negativas do stress no bem-estar do individuo, parece não reduzir aquela que é a sua experiência de stress. De facto, a autocompaixão permite que os estímulos stressores sejam reavaliados e vivenciados sob uma perspetiva mais positiva com base na autobondade, promovendo a aceitação da sua própria participação nesses eventos (Neely et al., 2009). Paralelamente, uma intervenção baseada na autocompaixão conduzida por Mosewich et al. (2013), concluiu que a promoção de autocompaixão parece reduzir os níveis de ruminação, autocriticismo e preocupação com o erro em atletas do sexo feminino. No entanto, a população que foi alvo de intervenção comportava apenas atletas com elevados níveis de autocriticismo do sexo feminino, o que não permite generalizar os resultados desta intervenção para outras populações. De facto, a amostra do presente estudo é maioritariamente constituída por jovens atletas do sexo masculino, o que poderá justificar os resultados obtidos. Por outro lado, alguns estudos encontraram que os atletas demonstram resistência à autocompaixão (e.g., Huysmans \& Clement, 2017; Reis et al., 2015), e acreditam que o autocriticismo é uma estratégia de regulação emocional crucial para o seu sucesso desportivo (Ferguson et al., 2015; Rodriguez \& Ebbeck, 2015; Sutherland et al., 2014), embora a literatura indique que o autocriticismo tem sido associado a indicadores negativos nos atletas, ao contrário da autocompaixão (Breines \& Chen, 2012). Ainda assim, muitos atletas temem que a autocompaixão possa induzir a complacência (Reis et al., 2015). Outro estudo conduzido, por Huysmans e Clement (2017), em uma amostra maioritariamente constituída por futebolistas adultos do sexo masculino sublinhou que esta população pode constituir-se como uma subcultura no desporto que parece ser ainda mais resistente à autocompaixão, e que a resistência do atleta à autocompaixão poderia ser fruto de autorrelatos imprecisos ou tendenciosos. Esta explicação pode suportar os nossos dados, uma vez que a amostra do presente estudo também é constituída maioritariamente por futebolistas do sexo masculino, apesar de serem adolescentes.

No sentido de analisar a hipótese principal, ou seja, se a perceção dos atletas acerca das atitudes críticas do treinador tem um efeito na ansiedade desportiva, através do mindfulness, autojulgamento e autocompaixão, foi realizada uma análise de trajetórias. No entanto, tendo em conta os resultados das correlações e regressão linear múltipla, o modelo testado não contemplou a variável autocompaixão. 0 modelo teórico testado apresentou uma excelente adequação aos dados e explicou $45 \%$ da variância da ansiedade desportiva. Os dados sugeriram que os atletas que percecionam mais atitudes críticas por parte do treinador tendem a apresentar maiores níveis de ansiedade desportiva, sendo esta relação totalmente mediada por menores níveis de mindfulness e maiores níveis de autojulgamento. Deste modo, os resultados do presente estudo parecem confirmar a hipótese de que as atitudes críticas do treinador têm um efeito na ansiedade desportiva dos atletas, através do mindfulness e do autojulgamento, mas não da 
autocompaixão. De facto, atletas que percecionam mais atitudes críticas por parte dos seus treinadores parecem internalizar este criticismo, revelando maiores níveis de autojulgamento, que corresponde a um mecanismo emocional maladaptativo para lidar com o erro e com o fracasso, o que parece estar associado a maiores níveis de ansiedade desportiva. Por outro lado, o mindfulness parece ser uma competência adaptativa para os atletas neste contexto, associando a menores níveis de ansiedade desportiva. Por outro lado, apesar de a autocompaixão se constituir uma estratégia adaptativa, neste estudo não revelou resultados significativos com as restantes variáveis. Dado que alguns estudos anteriores revelaram os benefícios da autocompaixão no contexto desportivo, seria importante que em estudos futuros fosse explorado o papel desta variável nos atletas.

Estes resultados deverão ser analisados tendo em consideração algumas limitações. Uma das limitações refere-se à natureza transversal do estudo, não permitindo retirar conclusões de causalidade. Assim, sugere-se que estudos futuros possam testar o presente modelo, recorrendo a um planeamento longitudinal ou experimental. Outra limitação encontra-se associada à situação pandémica da COVID-19 vivida aquando da recolha dos dados. Apesar de grande parte da recolha dos dados ter sido realizada presencialmente, outra parte foi realizada online, uma vez que houve uma suspensão da prática desportiva em Portugal, o que dificultou a adesão dos atletas ao estudo e, consequentemente, o tamanho reduzido da amostra. Neste sentido, seria ainda pertinente testar o presente modelo através de uma análise de equações estruturais completa de forma a obter resultados mais fidedignos. Paralelamente, o stress gerado pela pandemia COVID-19 (o que inclui a suspensão dos treinos) também poderá ter enviesado as respostas dos participantes. Por fim, a amostra do presente estudo foi composta por atletas da zona centro de Portugal, pelo que não é possível generalizar os resultados. Estudos futuros deverão explorar o papel destas variáveis não só em atletas de diferentes zonas do País, mas também de outras modalidades não contempladas nesta investigação.

Como pontos fortes do presente estudo é possível destacar a vertente inovadora do mesmo, que salientou não só o papel do treinador, mas também a importância de dois processos psicológicos na ansiedade desportiva, nomeadamente o papel do mindfulness e do autojulgamento. De facto, este estudo mostrou que a perceção dos atletas acerca de atitudes críticas por parte do treinador parece ser importante nos níveis de ansiedade desportiva, e especificamente no desenvolvimento de processos emocionais. Assim, treinadores que direcionam maioritariamente comentários críticos aos comportamentos e desempenho desportivo dos seus atletas e que menorizam os seus sentimentos parecem contribuir para níveis mais elevados de ansiedade desportiva. Assim, este estudo poderá ter implicações práticas importantes, alertando os treinadores para as consequências adversas de atitudes críticas e sublinhando a pertinência de adotarem atitudes mais compassivas e de suporte, as quais se têm revelado positivamente associadas a indicadores positivos nos atletas (Oliveira et al., 2021c). Além disso, este estudo parece sugerir a importância de promover estratégias adaptativas como o mindfulness nos jovens atletas. Assim, a presente investigação parece sugerir o desenvolvimento de programas de intervenção direcionados para jovens atletas que promovam o mindfulness enquanto estratégia adaptativa para lidar com os desafios do contexto desportivo, uma vez que esta capacidade de prestar atenção de uma forma particular ao momento presente e sem julgamento parece ser eficaz na ansiedade desportiva. De facto, intervenções baseadas no mindfulness têm demonstrado resultados promissores em jovens atletas (e.g., Bernier et al., 2014), no entanto, em Portugal, não existem intervenções direcionadas para esta população. Ainda assim, Carraça e colegas (2018) desenvolveram e conduziram um programa em Portugal direcionado para atletas 
adultos que revelou resultados promissores na saúde mental e desempenho desportivo de atletas adultos, o que salienta a necessidade e importância destes programas baseados no mindfulness em jovens. Por outro lado, o desenvolvimento destes programas também deverá ter em consideração a diminuição de atitudes de autodepreciação, de crítica negativa, de isolamento, comparação negativa perante o seu desempenho desportivo malsucedido, uma vez que este autojulgamento demonstrou uma associação com elevados níveis de ansiedade desportiva. De facto, a ansiedade desportiva é uma das principais causas do empobrecimento do gosto pela prática desportiva e do consequente abandono nas camadas jovens (Grossbard et al., 2009; Scanlan et al., 2005). Torna-se, assim, importante que estas variáveis possam ser alvo de investigação e intervenção, constituindo um contributo para o desporto em Portugal.

\section{Referências}

Bali, A. (2015). Psychological factors affecting sports performance. International Journal of Physical Education, Sports and Health, 1(6), 92-95. https://bit.ly/3042HJO

Baltzell, A., \& Akhtar, V. (2014). Mindfulness Meditation Training for Sport (MMTS) intervention: Impact of MMTS with Division I female athletes. The Journal of Happiness and Well-being, 2(2), 160-173. https://bit.ly/3243efv

Baltzell, A., Rothlin, P., \& Kentta, G. (2020). Self-compassion in sport for courage and performance. Em K. Henriksen, J. Hansen, \& C. Larsen (Eds.), Mindfulness and acceptance in sport (pp. 178-190). Routledge.

Bernier, M., Thienot, E., Pelosse, E., \& Fournier, J. (2014). Effects and underlying processes of a mindfulnessbased intervention with young elite figure skaters: Two case studies. The Sport Psychologist, 28, 302-315.

Breines, J., \& Chen, S. (2012). Self-Compassion Increases Self-Improvement Motivation. Personality and Social Psychology Bulletin, 38(9), 1133-1143. https://doi.org/cwz5

Brewin, C. R., Andrews, B., \& Furnham, A. (1996). Self-critical attitudes and parental criticism in young women. British Journal of Medical Psychology, 69(1), 69-78. https://doi.org/ft9n97

Bühlmayer, L., Birrer, D., Röthlin, P., Faude, O., \& Donath, L. (2017). Effects of mindfulness practice on performance-relevant parameters and performance outcomes in sports: A meta-analytical review. Sports Medicine, 47(11), 2309-2321. https://doi.org/gb4ffx

Carraça, B., Serpa, S., Rosado, A., \& Palmi, J. (2018). The Mindfulness-Based Soccer Program (MBSoccerP): Effects on elite athletes. Cuadernos de Psicología del Deporte, 18(3), 62-85. https://bit.ly/3wOjhcF

Côté, J., \& Fraser-Thomas, J. (2007). Youth involvement in sport. Em P. R. E. Crocker (Ed.), Sport psychology: A Canadian perspective (266-294). Pearson Prentice Hall.

Cruz, J., \& Gomes, A. (2007). Escala de Ansiedade no Desporto 2 - EAD-2. Manuscrito não publicado. Universidade do Minho.

Cunha, M., Galhardo, A., \& Pinto-Gouveia, J. (2013a). Child and Adolescent Mindfulness Measure (CAMM): Estudo das características psicométricas da versão portuguesa. Psicologia: Reflexão e Crítica, 26(3), 459-468. https://doi.org/g422

Cunha, M., Xavier, A., \& Castilho, P. (2016). Understanding self-compassion in adolescents: Validation study of the Self-Compassion Scale. Personality and Individual Differences, 93, 56-62. https://doi.org/f8cc2r

Cunha, M. Xavier, A., \& Vitória, I. (2013b). Avaliação da auto-compaixão em adolescentes: Adaptação e qualidades psicométricas da Escala de Auto-Compaixão. Revista de Psicologia da Criança e do Adolescente, 4(2), 95-117. https://bit.ly/3o3t4b0

Davis, L., \& Jowett, S. (2010). Investigating the interpersonal dynamics between coaches and athletes based on fundamental principles of attachment. Journal of Clinical Sport Psychology, 4(2), 112-132. https://doi.org/gjdwc3

Dehghani, M., Delbar Saf, A., Vosoughi, A., Tebbenouri, G., \& Ghazanfari Zarnagh, H. (2018). Effectiveness of the mindfulness-acceptance-commitment-based approach on athletic performance and sports competition anxiety: A randomised clinical trial. Electronic Physician, 10(5), 6749-6755. https://doi.org/10.19082/6749

Ferguson, L., Kowalski, K., Mack, D., \& Sabiston, C. (2014). Exploring self-compassion and eudaimonic wellbeing in young women athletes. Journal of Sport and Exercise Psychology, 36(2), 203-216. https://doi.org/f52rwk 
Ferguson, L., Kowalski, K., Mack, D., \& Sabiston, C. (2015). Self-compassion and eudaimonic well-being during emotionally difficult times in sport. Journal of Happiness Studies, 16(5), 1263-1280, https://doi.org/f7s8x6

Frazer, A. L., Fite, P. J., Stone, K. J., \& Clinkenbeard, J. (2018). Parental criticism moderates sibling influence on proactive and reactive aggression. Journal of Child and Family Studies, 27(12), 4025-4032. https://doi.org/gfp25m

Frentz, D., McHugh, T., \& Mosewich, A. (2019). Athletes' experiences of shifting from self-critical to selfcompassionate approaches within high performance sport. Journal of Applied Sport Psychology, 32(6) 15331571. https://doi.org/g423

Gaetano, R., Paloma, F., \& Gaetano, A. (2015). Anxiety in the youth physical and sport activity. Mediterranean Journal of Social Sciences, 6(3), 227-230. https://doi.org/g424

Gilbert, P., Clarke, M., Hempel, S., Miles, J. N. V., \& Irons, C. (2004). Criticising and reassuring oneself: An exploration of forms, styles and reasons in female students. The British Psychological Society, 43(1), 31-50. https://doi.org/bpgch9

Gilbert, P., \& Irons, C. (2009). Shame, self-criticism, and self-compassion in adolescence. Em N. Allen \& L. Sheeber (Eds.), Adolescent emotional development and the emergence of depressive disorders (pp. 195214). Cambridge University Press. https://doi.org/ctkdr6

Grossbard, J., Smith, R., Smoll, F., \& Cumming, S. (2009). Competitive anxiety in young athletes: Differentiating somatic anxiety, worry, and concentration disruption. Anxiety, Stress, \& Coping: An International Journal, 22(2), 153-166. https://doi.org/dvqqtw

Halamová, J., Kanovský, M., Gilbert, P., Troop, N. A., Zuroff, D. C., Hermanto, N., Petrocchi, N., SommersSpijkerman, M., Kirby, J., Shahar, B., Krieger, T., Matos, M., Asano, K., Yu, F., Basran, J., \& Kupeli, N. (2018). The factor structure of the forms of self-criticising/attacking \& self-reassuring scale in thirteen distinct populations. Journal of Psychopathology and Behavioral Assessment, 40(4), 736-751. https://doi.org/g425

Hasanah, U., \& Refanthira, N. (2020). Human problems: Competitive anxiety in sport performer and various treatments to reduce it. Advances in Social Science, Education and Humanities Research, 395, $144-148$. https://doi.org/g43d

Hu, L., \& Bentler, P. (1999). Cutoff criteria for fit indexes in covariance structure analysis: Conventional criteria versus new alternatives. Structural Equation Modelling: A Multidisciplinary Journal, 6(1), 1-55. https://doi.org/dbt

Huysmans, Z., \& Clement, D. (2017). A preliminary exploration of the application of self-compassion within the context of sport Injury. Journal of Sport \& Exercise Psychology, 39(1), 56-66. https://doi.org/gbmfw9

Josefsson, T., Ivarsson, A., Gustafsson, H., Stenling, A., Lindwall, M., Tornberg, R., \& Böröy, J. (2019). Effects of Mindfulness-Acceptance-Commitment (MAC) on sport-specific dispositional mindfulness, emotion regulation, and self-rated athletic performance in a multiple-sport population: An RCT study. Mindfulness, 10, 1518-1529. https://doi.org/gh8fw4

Jowett, S., \& Poczwardowski, A. (2007). Understanding the coach-athlete relationship. Em S. Jowette \& D. Lavallee (Eds.), Social psychology in sport (pp. 3-14). Human Kinetics.

Jowett, S., \& Nezlek, J. (2011). Relationship interdependence and satisfaction with important outcomes in coach-athlete dyads. Journal of Social and Personal Relationships 29(3), 287-301. https://doi.org/dnvrfr

Kabat-Zinn, J. (1994). Wherever you go, there you are: Mindfulness meditation in everyday life. Hyperion.

Kaufman, K., Glass, C., \& Arnkoff, D. (2009). Evaluation of Mindful Sport Performance Enhancement (MSPE): A new approach to promote flow in athletes. Journal of Clinical Sports Psychology, 3(4), 334-356. https://doi.org/10.1123/jcsp.3.4.334

Killham, M., Mosewich, A., Mack, D., Gunnell, K., \& Ferguson, L. (2018). Women athletes' self-compassion, selfcriticism, and perceived sport performance. American Psychological Association, 7(3), $297-307$. https://doi.org/gd3ngg

Kline, R. (2005). Principals and practice of structural equation modelling ( $2^{\mathrm{a}}$ ed.). Guilford Press.

Koestner, R., Zuroff, D., \& Powers, T. (1991). The family origins of adolescent self-criticism and its continuity into adulthood. Journal of Abnormal Psychology, 100(2), 191-197. https://doi.org/c2vbkx

Lee, K. H., Siegle, G. J., Dahl, R. E., Hooley, J. M., \& Silk, J. S. (2015). Neural responses to maternal criticism in healthy youth. Social Cognitive and Affective Neuroscience, 10(7), 902-912. https://doi.org/f72j5g

Machado, T., Paes, M., Alquiere, S., Osiecki, A., Lirani, L., \& Stefanello, J. (2016). Ansiedade estado précompetitiva em atletas de voleibol infanto-juvenis. Revista Brasileira de Educação Física e Esporte, 30(4), 1061-1067. https://doi.org/g426

Marôco, J. (2018). Análise estatística com o SPSS statistics ( $7^{\mathrm{a}}$ ed.). Report Number.

McLeod, B., Weisz, J., \& Wood, J. (2007). Examining the association between parenting and childhood depression: A meta-analysis. Clinical Psychology Review, 27(8), 986-1003. https://doi.org/b6xxzp 
Mosewich, A., Crocker, P., Kowalski, K., \& DeLongis, A. (2013). Applying self-compassion in sport: An intervention with women athletes. Journal of Sport \& Exercise Psychology, 35(5), 514-524. https://doi.org/f5gzv3

Mosewich, A., Kowalski, K., Sabiston, C., Sedgwick, W., \& Tracy, J. (2011). Self-compassion: A potential resource for young women athletes. Journal of Sport \& Exercise Psychology, 33(1), $103-123$. https://doi.org/10.1123/jsep.33.1.103

Neely, M., Schallert, D., Mohammed, S., Roberts, R., \& Chen, Y. (2009). Self-kindness when facing stress: The role of self-compassion, goal regulation, and support in college students' well-being. Motivation and Emotion, 33(1), 88-97. https://doi.org/cnzpsr

Neff, K. (2003). Self-compassion: an alternative conceptualisation of a healthy attitude toward oneself. Self and Identity, 2(2), 85-101. https://doi.org/dsthm2

Norman, L., \& French, J. (2013). Understanding how high performance women athletes experience the coachathlete relationship. International Journal of Coaching Science, 7(1), 3-24. https://bit.ly/30pJT7M

Oliveira, S., Trindade, I., Rosado, A., Cunha, M., \& Ferreira, C. (2021a). Development and initial validation of athletes' perceptions of coach-related critical attitudes scale. Current Psychology. https://doi.org/g427

Oliveira, S., Rosado, A., Cunha, M., \& Ferreira, C. (2021b). The compassionate coach scale as perceived by the athlete: development and initial validation in Portuguese athletes. International Journal of Sport and Exercise Psychology. https://doi.org/g428

Oliveira, S., Cunha, M., Rosado, A., \& Ferreira, C. (2021c). Compassionate coach and psychological quality of life in Portuguese athletes: effect of mediating variables. Journal of Clinical Sport Psychology. https://doi.org/10.1123/jcsp.2020-0058

Palmi, J., \& Solé, S. (2016). Intervenciones basadas en mindfulness (Atención Plena) en psicología del deporte. Revista de Psicología del Deporte, 25(1), 147-155https://bit.ly/306beMn

Pestana, M. H., \& Gageiro, J. N. (2008). Análise de dados para ciências sociais. A complementaridade do SPSS (5 $5^{\mathrm{a}}$ ed.). Edições Sílabo.

Piló, D. (2013). Autocriticismo, estima corporal e sintomas de perturbação do comportamento alimentar em adolescentes do sexo feminino, praticantes de ballet e andebol [Tese de mestrado, Instituto Superior Miguel Torga]. Repositório do Instituto Superior Miguel Torga. https://bit.ly/3kFiANX

Pinto-Gouveia, J., \& Xavier, A. (2010). O (in)sucesso na competição desportiva: a influência da aceitação e do auto-criticismo. Psychologica, 52(2), 361-386. https://doi.org/g429

Poczwardowski, A., Barott, J. E., \& Jowett, S. (2006). Diversifying approaches to research on athlete-coach relationships. Psychology of Sport and Exercise, 7(2), 125-142. https://doi.org/b68m59

Reis, N., Kowalski, K., Ferguson, L., Sabiston, C., Sedgwick, W., \& Crocker, P. (2015). Selfcompassion and women athletes' responses to emotionally difficult sport situations: An evaluation of a brief induction. Psychology of Sport and Exercise, 16(3), 18-25. https://doi.org/f6whr5

Ribeiro, C., Gomes, A., Simães, C., Resende, R., \& Moreira, D. (2016). Liderança, satisfação e perceção de rendimento desportivo: Estudo com atletas seniores. Journal of Sport Pedagogy \& Research, 2(1), $72-86$. https://bit.ly/3qdsObX

Rodriguez, M., \& Ebbeck, V. (2015). Implementing self-compassion strategies with female college gymnasts. Journal of Sport Psychology in Action, 6(1), 44-53. https://doi.org/g43b

Santos, A., Lofrano-Prado, M., Moura, P., Silva, E., Leão, A., \& Freitas, C. (2013). Ansiedade pré-competitiva em jovens atletas de nado sincronizado: uma análise à luz dos aspetos emocionais. Revista Da Educação Física/UEM, 24(2), 207-214. https://bit.ly/3wnCm5d

Scanlan, T., Babkes, M., \& Scanlan, L. (2005). Participation in sport: A developmental glimpse at emotion. Em J. L. Mahoney, R. W. Larson, \& J. S. Eccles (Eds.), Organised activities as contexts of development: Extracurricular activities, after-school and community programs (pp. 275-309). Lawrence Erlbaum Associates Publishers.

Scott-Hamilton, J., Schutte, N., \& Brown, R. (2016). Effects of a mindfulness intervention on sports-anxiety, pessimism, and flow in competitive cyclists. Applied Psychology: Health And Well-Being, 8(1), 85-103. https://doi.org/gdj4v6

Siekanska, M., Blecharz, J., \& Wojtowicz, A. (2013). The athlete's perception of coaches' behavior towards competitors with a different sports level. Journal of Human Kinetics, 39(1), 231-242. https://doi.org/g43c

Spínola, D. (2019). A relação entre a capacidade de mindfulness e a ansiedade competitiva dos atletas de halterofilismo e powerlifting [Tese de mestrado, Universidade Lusófona de Humanidades e Tecnologias]. Repositório Científico Lusófona. https://bit.ly/3ovTFh3

Sutherland, L., Kowalski, K., Ferguson, L., Sabiston, C., Sedgwick, W., \& Crocker, P. (2014). Narratives of young women athletes' experiences of emotional pain and self-compassion. Qualitative Research in Sport, Exercise and Health, 6(4), 499-516. https://doi.org/ggmv6c 
Tabachnick, B., \& Fidell, L. (2013). Using multivariate statistics (6 ${ }^{\mathrm{a}}$ ed.). Pearson.

Walton, C., Baranoff, J., Gilbert, P., \& Kirby, J. (2020). Self-compassion, social rank, and psychological distress in athletes of varying competitive levels. Psychology of Sport and Exercise, 50, 1-6. https://doi.org/gg2mnn Weinberg, R., \& Gould, D. 2017. Fundamentos da psicologia do esporte e do exercício. Artmed.

Wood, J., McLeod, B., Sigman, M., Hwang, W. C., \& Chu, B. (2003). Parenting and childhood anxiety: Theory, empirical findings, and future directions. Journal of Child Psychology and Psychiatry, 44(1), 134-151. https://doi.org/dmsw8f 